\section{$\mathrm{R}-\mathrm{CHOP}$ can be safely de-escalated in NHL}

Patients with aggressive $B$ cell non-Hodgkin lymphoma (B-NHL) typically receive six cycles of the R-CHOP regimen (comprising rituximab, cyclophosphamide, doxorubicin, vincristine and prednisone). Patients with a very favourable prognosis (defined by International Prognostic Index (IPI) risk factors) who receive $\mathrm{R}-\mathrm{CHOP}$ generally have favourable outcomes, including a 3-year progression-free survival (PFS) of approximately $95 \%$. However, this observation implies overtreatment of some patients. Now, new data from a phase III trial confirm that these patients can safely be spared two cycles of R-CHOP, and the associated adverse events.

A total of 592 patients $\leq 60$ years of age with very favourable-prognosis B-NHL were randomized (1:1) to receive either 6 cycles of R-CHOP, or 4 cycles of R-CHOP followed by two doses of rituximab. Non-inferiority, in terms of PFS, was the primary end point. At a median follow-up duration of 66 months, 3-year PFS was $96 \%$ among patients receiving 4 cycles of R-CHOP versus $94 \%$ in those receiving 6 cycles. This difference of $+3 \%$ demonstrates non-inferiority, according to the prespecified lower margin of $-5.5 \%$. Both groups had similarly high 3-year rates of disease remission ( $91 \%$ versus $92 \%$ ), event-free survival (89\% versus $89 \%$ ) and overall survival ( $99 \%$ versus $98 \%$ ).

Patients receiving four cycles of $\mathrm{R}-\mathrm{CHOP}$ had fewer nonhaematological $(1,036$ versus $1,280)$ and haematological (294 versus 426$)$ adverse events (AEs) of any grade, respectively; although the incidence of clinically AEs (48 versus 45) and secondary neoplasms (in $6 \%$ versus $5 \%$ of patients), respectively, were similar.

These findings confirm that patients with aggressive B-NHL with very favourable prognostic features can safely be spared two cycles of R-CHOP, and this will likely lead to improvements in quality of life, owing to fewer AEs. Whether patients $\geq 60$ years of age or with other specific less-favourable prognostic features in isolation can also receive fewer cycles of R-CHOP remains unclear.

Peter Sidaway

ORIGINAL ARTICLE Poeschel, V. et al. Lancet 394, 2271-2281 (2019)

\title{
AKT inhibition improves OS in TNBC
}

Chemotherapy is still the mainstay treatment for triple-negative breast cancer (TNBC) and patient outcomes remain poor. $\mathrm{PI} 3 \mathrm{~K}-\mathrm{AKT}$ signalling is often dysregulated in TNBC; now, new data from the randomized, double-blind, phase II PAKT trial support the addition of AKT inhibition to chemotherapy.

PAKT involved 140 women with untreated metastatic TNBC who were randomly assigned (1:1) to receive paclitaxel plus either the pan-AKT inhibitor capivasertib or placebo. Intermittent dosing of capivasertib (4 days on, 3 days off) was chosen over continuous daily dosing on the basis of data from phase I trials. The median progression-free survival (mPFS) duration was 5.9 months with capivasertib versus 4.2 months with placebo (1-sided $P=0.06$, meeting the predefined significance level of 1 -sided $P=0.10$ ). Only small, nonsignificant increases in the objective response rate (ORR, $34.8 \%$ versus $28.8 \%$ ), clinical benefit rate (CBR, 41.4 versus $37.1 \%$ ) and median duration of response ( $\mathrm{mDoR}, 7.6$ months versus 7.3 months) were observed. However, median overall survival (mOS) was prolonged by 6.5 months with capivasertib (19.1 months versus 12.6 months; 2 -sided $P=0.04$ ). The benefits of capivasertib seem to be limited mostly to patients with genetic alterations in PIK3CA, AKT1 or PTEN $(n=28)$. In this subgroup, the ORR, CBR, $\mathrm{mDoR}$, $\mathrm{mPFS}$ and $\mathrm{mOS}$ were $35.3 \%, 52.9 \%, 13.3$ months, 9.3 months and not reached, respectively, with capivasertib versus $18.2 \%, 27.3 \%$, 3.5 months, 3.7 months and 10.4 months with placebo.

The grade 3-4 adverse event rate was higher with capivasertib ( $54.4 \%$ versus $25.7 \%$ ). The excess toxicities were mainly limited to the known class effects of AKT inhibitors (such as diarrhoea, rash and hyperglycaemia) and were manageable.

These results are similar to those observed with ipatasertib, a different AKT inhibitor, in the LOTUS trial, thus further validating the approach of adding an AKT inhibitor to chemotherapy for TNBC.

\section{David Killock}

ORIGINAL ARTICLE Schmid, P. et al. Capivasertib plus paclitaxel versus placebo plus paclitaxel as first-line therapy for metastatic triple-negative breast cancer: the PAKT trial. J. Clin. Oncol. https://doi.org/10.1200/JCO.19.00368 (2019)

\section{Initial results with asciminib in CML}

Approximately half of all patients with BCR-ABL1 tyrosine-kinase inhibitor (TKI)-resistant chronic myeloid leukaemia (CML) have mutations in the ATP-binding site of the kinase. Now, the results of a phase I trial indicate that asciminib, a potent and specific TKI that mimics an autoinhibitory mechanism in ABL1 that is lost in the fusion protein, demonstrates activity in patients with relapsed or refractory CML.

This trial involved 150 patients with CML, of whom $70 \%$ had received $\geq 3 \mathrm{TKIs}$ and $31 \%$ had at least one mutation in the kinase domain of BCR-ABL1, most commonly T315l. Among 113 patients with chronic-phase CML and without a T315I mutation, a major molecular response (MMR, defined according to the International Scale) was achieved or maintained by 6 months in 37 of 99 patients (37\%) who could be evaluated, and by 12 months in 44 of 91 patients ( $48 \%$ ). The median duration of response (DOR) was $>61$ weeks.

By 12 months, 57 of 91 patients (63\%) had an improvement in their molecularresponse category. Among 28 patients with chronic-phase CML with a T315I mutation, an MMR was achieved in 4 of 17 patients $(24 \%)$ and maintained in 1 patient at 12 months, with a median DOR $>25$ weeks. By 12 months, 9 of 18 patients (50\%) had an improvement in their molecularresponse category. Among 9 patients with accelerated-phase CML, one had an MMR for $>11$ weeks.

Eight dose-limiting toxicities were reported. The most common adverse events (AEs) were asymptomatic elevations in serum lipase or amylase levels, rash, fatigue, nausea, headache and arthralgia; of these $\mathrm{AEs}$, lipase elevations were those with a higher incidence as a grade $3 \mathrm{AE}(10 \%)$. Pancreatitis occurred in 5 patients (grade $\geq 3 \mathrm{AE}$ in three of them), and resolved after treatment discontinuation.

These results demonstrate that asciminib is a potential treatment option for patients with CML that is relapsed and/or refractory to other TKIs. Several randomized controlled trials are underway, with results eagerly awaited.

Diana Romero

ORIGINAL ARTICLE Hughes, T. P. et al. Asciminib in chronic myeloid leukemia after ABL kinase inhibitor failure. N. Engl. J.Med. 381, 2315-2326 (2019) 